\title{
Das Institut für Sächsische Geschichte und Volkskunde in Dresden Bericht für das Jahr 2017
}

von

\author{
WINFRIED MÜLLER
}

Mit dem Tätigkeitsbericht für 2017 wird Rechenschaft über ein Arbeitsjahr abgelegt, in dem nicht nur die projektbezogene wissenschaftliche Arbeit im Zentrum stand, sondern in dem das ISGV im 20. Jahr seines Bestehens zugleich einer umfassenden Bestandsaufnahme unterzogen wurde. Zum einen wurde die Prüfung der Ausgabe- und Verwaltungspraxis des Instituts durch den sächsischen Rechnungshof mit positivem Ergebnis zum Abschluss gebracht. Anregungen und Hinweise wurden in Gesprächen mit dem zuständigen Referat des Sächsischen Staatsministeriums für Wissenschaft und Kunst, dem Kuratorium und der Innenrevision der TU Dresden besprochen und umgesetzt. Hier ist vor allem anzuerkennen, dass der Forderung des Rechnungshofs an den Zuwendungsgeber, für eine angemessene Ausfinanzierung des Instituts zu sorgen, im Doppelhaushalt 2017/18 entsprochen wurde.

Zum anderen stand das Jahr 2017 im Zeichen der wissenschaftlichen Bilanzierung. Eine Evaluierungskommission, die sich sowohl aus den Mitgliedern des Wissenschaftlichen Beirats als auch aus externen Gutachtern zusammensetzte, legte auf der Grundlage eines durch das ISGV ausführlich beantworteten Fragenkatalogs sowie einer Begehung, die am 30./31. März 2017 durchgeführt wurde, am 16. Mai 2017 ihren Abschlussbericht vor. Er attestierte dem Institut, „sowohl national wie international in der wissenschaftlichen Öffentlichkeit hervorragend aufgestellt" zu sein, das ISGV genieße „im In- und Ausland eine hervorragende wissenschaftliche Reputation“. Neben dieser grundsätzlichen Einschätzung, die erfreut zur Kenntnis genommen wurde, enthielt der Bericht konstruktive Anregungen sowohl für die weitere wissenschaftliche Arbeit als auch für notwendige Anpassungen im Stellenplan, über die in den Gremien des ISGV diskutiert und mit deren Umsetzung noch 2017 begonnen wurde.

Auch im Sinne einer Bilanz war schließlich die aus Anlass des 20-jährigen Institutsjubiläums am 19./20. Oktober 2017 durchgeführte Tagung „Landesgeschichte und Volkskunde in der DDR und in den neuen Ländern“ zu verstehen, deren Vorträge in den „Blättern für deutsche Landesgeschichte“ erscheinen werden. 20 Jahre nach seiner Gründung thematisierte das ISGV damit Traditionen, Brüche und Kontinuitätslinien der Landes- und Regionalgeschichte sowie der Volkskunde in Sachsen und in den sogenannten neuen Ländern. Gleichfalls dem Institutsjubiläum verpflichtet war der zum Jahresabschluss vorgelegte Band „Das Institut für Sächsische Geschichte und Volkskunde 1997-2017“, der ausführlich Projekte und Themenfelder vorstellt und die Publikations- und Tagungsaktivitäten des ISGV dokumentiert. Einen prominenten Platz nahm dabei im Luther-Jahr 2017 die Reformation ein. Tagungen, darunter eine in Verbindung mit dem Historischen Institut der Tschechischen Akademie der Wissenschaften durchgeführte Konferenz in Prag, zahlreiche Fachvorträge sowie Buchpräsentationen einschlägiger Publikationen des ISGV in Dresden und Leipzig dokumentierten die Leistungskraft des Instituts auf dem für die Sächsische Landesgeschichte zentralen Forschungsfeld der Reformationsgeschichte. 
Daneben ging natürlich die wissenschaftliche Alltagsarbeit unvermindert weiter, indem zum einen die Langzeitprojekte wie „Sächsische Biografie“, „Codex diplomaticus Saxoniae“, „Fürstinnenkorrespondenzen der Reformationszeit“, „Visuelle Quellen zur Volkskultur in Sachsen“"und "Lebensgeschichtliches Archiv für Sachsen“ kontinuierlich weiterverfolgt wurden. Mittelfristig angelegte Arbeitsvorhaben wie die Entwicklung Plauens zur Großstadt in der Phase der Hochindustrialisierung oder das über Drittmittel finanzierte Kontaktzonen-Projekt über kulturelle Praktiken im deutsch-tschechisch-polnischen Dreiländereck wurden ganz oder teilweise abgeschlossen. Neue Projekte zur Finanz- und Geldgeschichte Sachsens im 18. Jahrhundert, zur Denkmalkultur in Sachsen 1871 bis 1933, zur Wahrnehmung und Darstellung von Kriminalität im sächsisch-polnischen Grenzgebiet oder die Digitalisierung des Nachlasses von Adolf Spamer kamen hinzu. Vom letztgenannten Projekt werden wichtige Impulse für die Erschließung und analytische Auswertung der volkskundlichen Nachlässe und Sammlungen des ISGV ausgehen, da es als Teil des vom SMWK geförderten Verbundprojekts „Virtuelle Archive für die geisteswissenschaftliche Forschung“ die Vernetzung mit anderen Instituten und die Koordinierung digitaler Dokumentationsstrategien voranbringen wird.

Die hier nur exemplarisch aus dem breiten Arbeitsprogramm beider Bereiche herausgegriffenen Projekte werden ergänzt durch eine umfangreiche Vortrags-, Beratungs- und Publikationstätigkeit. Was die Beratungsaktivitäten betrifft, so brachte das ISGV 2017 seine Expertise u. a. im Wissenschaftlichen Beirat zur 4. Sächsischen Landesausstellung, die 2020 der Industriekultur gewidmet sein wird, ebenso ein wie bei der Vorbereitung des Plauener Stadtjubiläums 2022. Im Publikationssegment ergab sich 2017 im Bereich der Printmedien für die Reihen „Bausteine“ und „Spurensuche“ sowie für die Zeitschrift „Volkskunde in Sachsen“ die Notwendigkeit eines Verlagswechsels, der reibungslos verlaufen ist. Im Bereich der Internet-Publikationen und des Datenmanagements konnte auch vor dem Hintergrund einer personellen Neustrukturierung der gewohnt hohe Standard gehalten werden, allerdings reichen die Arbeitskapazitäten für die Bewältigung der stetig wachsenden Aufgaben bereits jetzt kaum mehr aus. Hier steht das ISGV vor großen Herausforderungen, die auch Gegenstand methodischer Reflexion sein müssen. Das ISGV wendet sich deshalb mit der 2017 konzipierten Tagung „Forschungsdesign 4.0 - Datengenerierung und Wissenstransfer in interdisziplinärer Perspektive“ grundsätzlichen Fragen der wissenschaftlichen Praxis im digitalen Zeitalter zu. Kernaufgabe bleibt freilich die landesgeschichtliche und volkskundliche Grundlagenforschung zu Sachsen und seinen Nachbarregionen, der sich die Mitarbeiterinnen und Mitarbeiter des Instituts in einer von der Evaluierungskommission ausdrücklich gewürdigten offenen Arbeitsatmosphäre auch im dritten Jahrzehnt der Institutsgeschichte mit hohem Engagement stellen werden.

\section{Forschungsprojekte 2017}

\section{Gemeinsame Projekte der Bereiche Geschichte und Volkskunde}

Schriften zur sächsischen Geschichte und Volkskunde; Quellen und Materialien zur sächsischen Geschichte und Volkskunde; Bausteine zur sächsischen Geschichte und Volkskunde; Spurensuche. Geschichte und Kultur Sachsens. Projektbearbeitung: Direktorium, Bereichsleiterinnen, wissenschaftliche Mitarbeiter.

Erinnerungslandschaften: Transformationsprozesse im ländlichen Raum Ostdeutschlands - öffentliche Diskurse und individuelle Wahrnehmungen (von 1945 bis in die Gegenwart). Projektbearbeitung: Henrik Schwanitz. 


\section{Projekte des Bereichs Geschichte}

Sächsische Biografie. Projektbearbeitung: Martina Schattkowsky, Frank Metasch, Henrik Schwanitz.

Fürstinnenkorrespondenzen der Reformationszeit: Briefedition der Herzogin Elisabeth von Sachsen, Band 3. Projektbearbeitung: Jens Klingner.

Codex diplomaticus Saxoniae (CDS). Die Papsturkunden für sächsische Empfänger; Das Urkundenbuch der Stadt Dresden. Projektbearbeitung: Enno Bünz, Christian Schuffels, Ulrike Siewert, Stefan Petersen.

Sächsisches Klosterbuch. Klöster, Stifte und Komtureien in Sachsen vor der Reformation. Projektbearbeitung: Enno Bünz, Sabine Zinsmeyer.

Für Gott und Vaterland. Patriotismus und Militärdienst in Sachsen 1806 bis 1866/67. Projektbearbeitung: Torsten Schwenke.

Finanz- und Geldgeschichte Sachsens im 18. Jahrhundert. Projektbearbeitung: Frank Metasch.

Herzogin Elisabeth von Sachsen. Biografie einer Reformationsfürstin. Projektbearbeitung: Jens Klingner.

Von der Natur gerahmt. Die Idee der „natürlichen Grenzen“ als Identitätsressource um 1800. Projektbearbeitung: Henrik Schwanitz.

Neues Archiv für sächsische Geschichte. Projektbearbeitung: Karlheinz Blaschke, Enno Bünz, Winfried Müller, Martina Schattkowsky, Uwe Schirmer (alle Herausgeber), Frank Metasch (Schriftleitung), Jens Klingner (Rezensionen), Anne Reim (Projektmitarbeit).

Zwischen Migration und Assimilation. Adel im sächsisch-böhmischen Grenzraum (16./17. Jahrhundert). Projektbearbeitung: Martin Arnold.

\section{Projekte des Bereichs Volkskunde}

Lebensgeschichtliches Archiv für Sachsen. Projektbearbeitung: Sönke Friedreich, Ira Spieker, Claudia Pawlowitsch, Nick Wetschel.

Visuelle Quellen zur Volkskultur in Sachsen. Das Bildarchiv des ISGV. Projektbearbeitung: Andreas Martin, Michael Schmidt, Hendrik Keller, Daniel Geißler, Jürgen Ruddat.

Kontaktzonen. Kulturelle Praktiken im deutsch-tschechisch-polnischen Grenzraum. Projektbearbeitung: Sarah Kleinmann, Arnika Peselmann, Ira Spieker.

An der Elbe. Das Leben mit dem Fluss. Projektbearbeitung: Andreas Martin.

Erinnern an die Arbeit im Kollektiv. Brigadeleben in der DDR und seine postsozialistischen Tradierungen. Projektbearbeitung: Merve Lühr. 
Zwischen Aufstieg und Krise. Städtische Identität und Selbstwahrnehmung in Plauen, 1880-1933. Projektbearbeitung: Sönke Friedreich.

Umstrittene Memoriale. Das „Zeitalter des Denkmals“ in Sachsen, 1871-1933. Projektbearbeitung: Sönke Friedreich.

Neue Sichtweisen. Zum Aufleben einer Aussichtsturm-Begeisterung. Projektbearbeitung: Andreas Martin.

Erschließung und Digitalisierung des Nachlasses Adolf Spamer. Projektbearbeitung: Nadine Kulbe, Hans Oppitz, Antje Reppe.

Grenzfälle. Wahrnehmung und Darstellung von Kriminalität und Devianz im deutschpolnischen Grenzgebiet seit 1949. Projektbearbeitung: Sarah Kleinmann.

Bildsehen - Bildhandeln. Die Freiberger Fotofreunde als Community of Visual Practice. Projektbearbeitung (Antrag): Ira Spieker, Nadine Kulbe, in Kooperation mit Torsten Näser vom Institut für Kulturanthropologie/Europäische Ethnologie, Göttingen.

Künstlersteinzeichnungen für Haus und Schule. Projektbearbeitung: Winfried Müller, Michael Schmidt (technische Umsetzung).

Jahrbuch „Volkskunde in Sachsen“. Projektbearbeitung: Ira Spieker, Sönke Friedreich, Mitarbeitende des Bereichs Volkskunde.

\section{Ausstellungen und Ausstellungskonzeptionen}

Verwoben. Geschichten in der Grenzregion. Verantwortlich: Ira Spieker.

Zwischen Tradition und Modernität - Zeugnisse sächsischer Adelskultur (Arbeitstitel). Verantwortlich: Martina Schattkowsky.

\section{Tagungen und Workshops}

Die industrielle Stadt. Lokale Repräsentationen von Industriekultur im urbanen Raum seit dem ausgehenden 19. Jahrhundert. Kolloquium des ISGV in Kooperation mit dem Sächsischen Staatsarchiv - Staatsarchiv Chemnitz, Chemnitz, 15./16. Juni 2017. Verantwortlich: Sönke Friedreich.

Adel und Reformation. Tagung des ISGV in Kooperation mit den Staatlichen Schlössern, Burgen und Gärten Sachsen gGmbH und dem Evangelischen Zentrum Ländlicher Raum, Heimvolkshochschule Kohren-Sahlis, Schloss Gnandstein, 7./8. September 2017. Verantwortlich: Martina Schattkowsky.

Johann Tetzel und der Ablass. Bilanz und Perspektiven. Workshop des ISGV, Leipzig, 8. Oktober 2017. Verantwortlich: Enno Bünz. 
Landesgeschichte und Volkskunde in der DDR und in den neuen Ländern. 20 Jahre Institut für Sächsische Geschichte und Volkskunde | 44. Tag der Landesgeschichte. Tagung des ISGV in Zusammenarbeit mit dem Gesamtverein der deutschen Geschichts- und Altertumsvereine, Dresden, Sächsische Landesbibliothek - Staats- und Universitätsbibliothek, 19./20. Oktober 2017. Verantwortlich: Winfried Müller, Klaus Neitmann (Potsdam).

„Kontaktzonen“ und Grenzregionen. Aktuelle kulturwissenschaftliche Perspektiven/ „Contact Zones“ and Border Regions. Current Perspectives in Cultural Sciences. Tagung des ISGV in Kooperation mit dem Sächsischen Staatsarchiv - Hauptstaatsarchiv Dresden, Dresden, 23./24. November 2017. Verantwortlich: Sarah Kleinmann, Arnika Peselmann.

Reformation als Kommunikationsprozess: Böhmische Kronländer - Sachsen - Mitteleuropa. Internationale Tagung in Kooperation mit dem Historischen Institut der Tschechischen Akademie der Wissenschaften in Prag und dem Lehrstuhl für Geschichte der Frühen Neuzeit der Technischen Universität Dresden, Prag, 28. bis 30. November 2017. Verantwortlich: Martin Holý, Petr Hrachovec, Jiří Just (Prag), Winfried Müller, Martina Schattkowsky, Gerd Schwerhoff (Dresden).

\section{Publikationen}

Schriften zur sächsischen Geschichte und Volkskunde, hrsg. von Enno Bünz, Winfried Müller, Martina Schattkowsky, Ira Spieker. Leipzig: Leipziger Universitätsverlag:

Band 56: KATRIn Lehnert, Die Un-Ordnung der Grenze. Mobiler Alltag zwischen Sachsen und Böhmen und die Produktion von Migration im 19. Jahrhundert, 2017.

Band 57: Sönke Friedreich, Der Weg zur Großstadt. Stadtentwicklung, bürgerliche Öffentlichkeit und symbolische Repräsentation in Plauen (1880-1933), 2017.

Quellen und Materialien zur sächsischen Geschichte und Volkskunde, hrsg. von Enno Bünz, Winfried Müller, Martina Schattkowsky, Ira Spieker. Leipzig: Leipziger Universitätsverlag:

Band 3.2: Jens Klingner (Hg.), Die Korrespondenz der Herzogin Elisabeth von Sachsen, Zweiter Band: Die Jahre 1533 und 1534, 2016.

Bausteine aus dem Institut für Sächsische Geschichte und Volkskunde. Kleine Schriften zur sächsischen Geschichte und Volkskunde, hrsg. von Enno Bünz, Winfried Müller, Martina Schattkowsky, Ira Spieker. Leipzig, Leipziger Universitätsverlag:

Band 37: Wolfgang Hesse/Holger Starke (Hg.), Arbeiter | Kultur | Geschichte. Arbeiterfotografie im Museum, 2017.

Spurensuche. Geschichte und Kultur Sachsens, hrsg. von Enno Bünz, Winfried Müller, Martina Schattkowsky, Ira Spieker. Dresden, Sandstein-Verlag:

Band 7: Institut für Sächsische Geschichte und Volkskunde 1997-2017, hrsg. vom Institut für Sächsische Geschichte und Volkskunde, Redaktion: Winfried Müller und Daniel Geißler, redaktionelle Mitarbeit: Susanne Müller, 2017. 
Neues Archiv für Sächsische Geschichte, hrsg. von Karlheinz Blaschke, Enno Bünz, Winfried Müller, Martina Schattkowsky, Uwe Schirmer. Redaktion: Frank Metasch (Schriftleitung), Jens Klingner (Rezensionen), Band 88 (2017). Neustadt a. d. Aisch, Verlag Ph. C. W. Schmidt.

Volkskunde in Sachsen. Jahrbuch für Kulturanthropologie, hrsg. vom Institut für Sächsische Geschichte und Volkskunde. Schriftleitung: Ira Spieker, Sönke Friedreich, Band 29 (2017). Weimar, Jonas Verlag.

\section{Online-Publikationen}

Lebensgeschichtliches Archiv für Sachsen. Verantwortlich: Ira Spieker, Michael Schmidt (technische Umsetzung), URL: http://lga.isgv.de.

Visuelle Quellen zur Volkskultur in Sachsen. Das Bildarchiv des ISGV. Verantwortlich: Andreas Martin, Michael Schmidt, Hendrik Keller (technische Umsetzung), URL: http://bild.isgv.de/.

Kontaktzonen. Begegnungen im Grenzgebiet. Verantwortlich: Sarah Kleinmann,

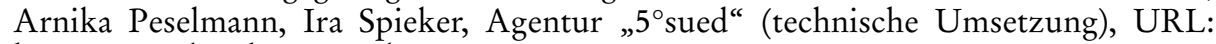
http://www.bordernetwork.eu.

Digitales Historisches Ortsverzeichnis von Sachsen (DHOV). Verantwortlich: Henrik Schwanitz, Michael Schmidt (technische Umsetzung), URL: http://hov.isgv.de/orte/.

Repertorium Saxonicum. Verantwortlich: Jens Klingner, Michael Schmidt (technische Umsetzung), URL: http://www.isgv.de/repsax/.

Der Codex diplomaticus Saxoniae im Internet. Verantwortlich: Christian Schuffels, Michael Schmidt (technische Umsetzung), URL: http://www.isgv.de/codex/.

Sächsische Gerichtsbücher. Verantwortlich: Volker Jäger, Martina Schattkowsky, Michael Schmidt (technische Umsetzung), URL: http://www.saechsische-gerichts buecher.de/projekt/.

Sächsische Biografie. Verantwortlich: Martina Schattkowsky, Michael Schmidt (technische Umsetzung), URL: http://saebi.isgv.de/.

Sachsen.digital: Interdisziplinäre Wissensplattform zur Geschichte, Kultur und Landeskunde Sachsens, gemeinsames Internetportal der Sächsischen Landesbibliothek Staats- und Universitätsbibliothek Dresden und des ISGV. Verantwortlich: Martina Schattkowsky, Ira Spieker, Michael Schmidt, SLUB (technische Umsetzung), URL: https://sachsen.digital/. 